\title{
On Primary Mathematics Teaching from the Perspective of Performance Technology
}

\section{Sang Shuangshuang}

Co-Innovation Center for Educational Informatization and Balanced Development of Basic Education, Central China Normal University, Wuhan, China

\section{Email address:}

1085181017@qq.com

\section{To cite this article:}

Sang Shuangshuang. On Primary Mathematics Teaching from the Perspective of Performance Technology. Science Discovery. Vol. 5, No. 6, 2017, pp. 434-437. doi: 10.11648/j.sd.20170506.17

Received: September 24, 2017; Accepted: October 5, 2017; Published: November 21, 2017

\begin{abstract}
In the mathematics teaching of primary school in China, the teaching of teachers makes students only solve the problems in books, but not solve similar problems in real life. The students lack of life common sense and the knowledge of the application. They have no sensitivity to mathematical knowledge in real life and can not apply the knowledge in practice. The problem is worthy of the attention of teachers. This paper will examine the mathematics teaching of primary school from the perspective of performance technology, analyze the existing problems and put forward corresponding solutions.
\end{abstract}

Keywords: Performance Technology, Primary Mathematics, Mathematics Teaching

\section{从绩效技术的角度审视小学数学教学}

桑双双

信息化与基础教育均衡发展协同创新中心, 华中师范大学, 武汉, 中国

邮箱

1085181017@qq.com

摘要: 在中国小学数学教学中, 教师的课堂教学使得学生只会解决书本上的问题, 而不会解决实际生活中的类似问 题。缺乏相应的生活常识和对所学知识的实地应用, 对实际生活中出现的数学知识没有一定的敏感性, 不能将课堂上 所学习到的知识进行实际的应用和迁移。这种问题是值得引起教师重视的。本文将从绩效技术的角度审视小学的数学 教学, 分析其中存在的问题并提出相应的解决对策。

关键词: 绩效技术, 小学数学, 数学教学

\section{1. 引言}

社会的不断发展, 对教师和学生都提出了新的要求: 教师要转变自己的教育理念, 改进自己原来习以为常的 教学方法、教学行为和教学手段, 重新认识和确立自己 的角色, 改变课堂教学模式, 提高自己的教育教学水平; 学生要具备创新思维, 勇于发现新的东西, 能够将知识 和实践相结合, 拥有较强的动手实践能力, 善于发现问
题、解决问题并提出自己的想法。只有这样才能逐步培 养学生的创新素质, 使其适应当前信息社会的发展潮流, 从而更好的推动社会的发展。众所周知, 数学教育的目 的不仅仅是使学生获得适应未来社会生活和进一步发展 所必需的重要数学知识和基本的问题解决方法, 更重要 的是能够运用数学知识解决实际生活中的问题。如今, 
问题解决教学模式逐渐得到了广大教师的青睐, 教师要 把握好数学学科的特点与小学生的学习规律, 引导学生 自主思考、分析、假设并验证问题, 最终真正理解和吸 收知识。文章将从绩效技术的角度重新对小学数学教学 进行相关分析。

\section{2. 小学数学的教学现状}

目前, 中国小学数学的教学情况存在以下几个问题:

\section{1. 教学形式单一，效率不高}

教师教学采用的多是传统的讲授式课堂教学模式, 教师为课堂的主体, 偏重知识性提问, 忽略学生的参与 和数学能力的培养等诸多问题, 学生的积极性不高, 只 是机械地记忆老师所讲的方法和内容, 缺乏自己的独立 思考, 更不用说能有效地将所学知识应用于实际生活中。 长此以往, 学生会逐渐失去对学习数学的兴趣和热情, 更糟糕地是这种教学方式会逐渐泯灭学生的学习天赋以 及独特的数学思维。

\section{2. 讲授内容与实际生活脱节}

数学家华罗庚曾经说过: “数学教学必须从学生熟悉 的生活情景和感兴趣的事情中提供观察和操作的机会, 使他们感受到数学就在身边, 感受到数学的趣味和作用, 对数学产生亲切感......”。当然, 这种描述是对数学教学 的最好阐述, 如果教师能做到, 那必然是一堂成功的教 学。然而在实际的教育教学中, 教师过分强调理论知识 的学习和记忆, 过分强调自己的“教”, 忽视学生的“学”, 忽视学生的主体地位; 同时教师讲授的内容基本都是照 本宣科, 没有自己的创新。信息化时代, 虽然教师可以 利用信息手段进行教学, 以此引发学生的学习兴趣, 但 并没有从根本上解决问题。学生的学习只停留在理论层 面, 没有生活方面的经验, 也没有实践的参与, 使得知 识与生活脱离, 理论与实践脱离。因此, 作为小学数学 教师, 有责任给学生创造一个熟悉的和较生活化的数学 学习环境, 使学生产生学习的兴趣并能感受到学习的快 乐, 在这种愉快轻松的氛围中进行探索性的学习, 将学 生难以理解的事物转化成学生所熟知的形象化事物, 这 种将数学知识生活化, 将数学与实际生活联系起来的一 种教学方式, 能够让学生更容易建构自己的数学知识体 系, 让他们懂得其实数学就在自己身边, 就在自己现实 的生活学习中。

\section{3. 评价体系不健全}

教学过程中, 评价和反馈是必不可少的。目前很多 教师的教学效果评价方式比较单一, 只重结果不重过程, 一份试卷便是教师检验学生学习效果的试金石。在这样 一种以分数定优劣的评价中, 教师只注重学生的最终分 数: 分数高, 就果断地认为学生学得好, 知识掌握牢靠; 分数低, 就断然认为学生学得差, 知识掌握不牢或没掌 握。这种以分数作为唯一的评价标准中, 教师对此过于 依赖而失去了自己的思考和判断, 忽略了那些分数高的
学生是不是因为之前做过这样的题记住了答案, 还是确 实会做这些题。如果教师不探究学生的学习过程, 就贸 然以成绩作为评价标准, 很可能会伤害那些爱学习的学 生, 也会伤害那些不善于学习但却有其他特长的学生。 因此只有单一的评价, 没有注重学生个性、特长、兴趣 的多元评价, 把所有学生都推向同一个跑道是不科学的, 是不利于学生的成长和发展的。

由上可知, 目前中国小学数学教学中的教学方式上 还存在不足, 在教学内容和评价上也存在一些缺陷。究 其原因, 是对教学的绩效特性还没有认清。因此, 对于 小学数学的教学有必要从绩效技术的角度对其进行重新 的审视和定位。

\section{3. 绩效技术}

\section{1 . 绩效技术}

绩效技术，又称为人类绩效技术 $[1]$, 起源于 20 世纪 60 年代末与 70 年代初的教学系统设计, 是教学系统设计 发展的产物, 其创始人是美国管理学家德鲁克（Peter F. Druker）。随着时代的发展, 人的智力资源成为信息社会 里最主要的资源, 而开发这种资源的绩效技术也就成为 了最主要的技术。

目前，对于绩效技术还没有统一的定义, 本文引用上 海外国语大学张祖忻教授关于绩效技术的界定：绩效技 术是运用分析、设计、开发、实施和评价的系统方法来 提高个人和组织机构工作业绩的研究领域 [2]。绩效技术 的核心是人, 它关心的是如何提高和保持人的生产力与 竞争能力。绩效技术的目标是实现行为者或员工以及系 统有价值的发展。因此, 人类绩效技术是一种提高生产率 和竞争能力的系统方法, 是一种解决问题的策略。目前, 当人们谈到绩效技术时, 绝大多数都认为绩效是应用在 企业培训中的, 往往忽略了绩效在教育教学活动中的应 用研究。其实从历史的角度看, 绩效技术起源于程序教 学和教学系统设计, 后来在企业培训领域得到了发展 [3], 教育和绩效技术有着巨大的联系。人类生存所需的完整 资源系统不仅需要一个完善的学习系统, 更需要关注绩 效。

总而言之, 绩效技术是为了达到理想的绩效水平而 采取的一种系统方法, 它通过相应的干预措施来缩小目 标绩效与当前绩效之间的差距。在教育教学中，绩效技 术把理想教学情况与现实情况进行对比分析, 找到其中 存在的差距, 即绩效差距, 然后选择合适的干预措施来 尽可能地缩小绩效差距, 最终实现理想的教学。

\section{2. 绩效技术过程模型}

绩效技术模型是为了揭示工作环境的复杂性和所有 要素之间的相互影响, 从而为绩效技术从业人员指明如 何在工作中提高绩效的操作步骤[4]。绩效技术过程模型 借鉴了教学系统化设计的经典模型, 即ADDIE模型。利 用绩效技术的一般过程如图1[5]所示。 


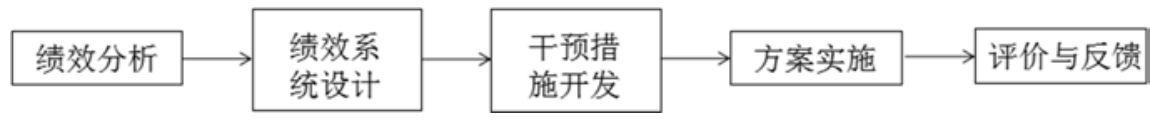

图1 绩效技术过程框架图。

从上图可以看出绩效技术过程与教学系统化设计过 程非常相似, ADDIE模型主要是从分析、设计、开发、 实施与评价进行教学系统设计, 而绩效技术过程模型则 从绩效分析、绩效系统设计、干预措施开发、方案实施 以及最后的评价与反馈进行问题的解决。在具体的教育 教学中, 关注教学绩效问题, 并运用绩效技术进行问题 的解决将对教学具有促进重要的促进作用。

\section{4. 绩效技术视野下小学数学的教学分析}

绩效技术是一种提高绩效的系统过程, 如果将“提高 绩效” 以一种更具普遍意义的话语来表述, 可以称之为一 个问题, 即绩效技术是用以解决某组织或个体的绩效要 优于现有状况这一问题的系统方法 [6]。这种系统方法是 可以根据问题及实际情况进行调整的。而绩效技术的最 大价值正是由于它的实践性和可造型, 为了将绩效技术 的基本过程思想应用于提高小学数学的教学中, 本文结 合小学数学的教学情况, 得出了人类绩效技术在教学实 践中的操作模型, 如图2所示。

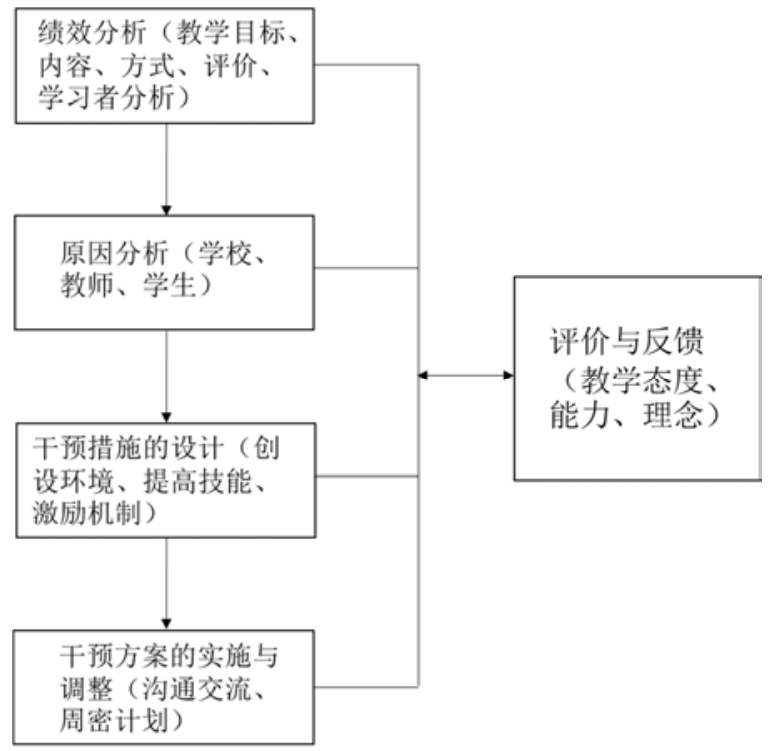

图2 教学的绩效技术实践结构图。

\section{1. 教学绩效分析}

绩效分析的目的是确定目前所实现的绩效状态与期 望绩效状态之间的差距, 以便为缩小这一差距应采取的 措施提供依据[7]。针对小学数学教学的实际, 绩效分析 将主要针对授课教师的教学目标、教学内容、教学方式、 教学评价, 以及学习者的学习偏好和认知水平等方面进 行系统的分析, 确定当前的绩效状态, 找出其中存在的 问题。

\section{2. 教学原因分析}

原因分析是在绩效分析的基础上进行的, 它主要是 寻找绩效差距产生的原因, 为下一步干预措施的设计和 实施提供依据。绩效技术的思想使得教学设计者应该从 更加宏观的角度考虑问题, 当存在绩效差距时, 需要进 行全面而宏观地分析, 寻找差距的真正原因, 而不是直 接归因于教师的教学。

在该模型中, 本文将从学校、教师和学生三个方面 进行分析。在学校方面, 教学政策指导不力、对教师的 绩效考核不完善、激励机制不健全等都会引起教学差距; 教师方面, 教师本身的教学知识与学识、教学素质与能 力、教学经验与技巧等方面的缺失也会导致教学质量的 下降; 学生方面, 缺乏学习的兴趣、缺乏自主学习的意 识和能力、缺乏明确的学习目标同样也是教学差距产生 的原因。当发现学生的学习效果不理想时, 就需要对教 师的教学进行原因分析, 确定引起教学差距的真正原因。

\section{3. 教学干预措施的设计}

干预措施即具体的实施方法。它是在确定了绩效差 距和原因的基础上进行的, 根据产生绩效差距的原因, 有针对性的选择相应的干预措施, 设计可行的干预方案。 在绩效技术指导下, 教师教学的干预措施可以是创设良 好的活动环境、提高教师的教学技能、鼓励教师实施活 动教学, 促进学生的协作学习和探究学习, 使得学生从 生活中发现问题、探索问题, 最终获取知识。

\section{4. 教学干预方案的实施与调整}

干预意味着改变, 改变必然需要学校领导及教师们 的支持与参与, 那么阻力也必然来源于他们, 或许是因 为利益冲突, 或许是因为固有的传统观念和行为习惯, 也或许是因为不信任等等。因此, 在进行干预措施实施 时, 需要考虑到所有可能遇到的问题, 针对不同的问题 做好不同的应对措施。做好周密的计划, 应对每个阶段 的突发状况。而调整是针对下面的评价反馈阶段进行的。

\section{5. 教学评价与反馈}

根据绩效技术理论, 教学的反馈与评价贯穿于整个 绩效过程。通过评价分析可以确定干预措施的成功与否, 反馈其中的问题, 以便对整个绩效过程进行及时地调整。 在此模型中, 教学评价为教学干预措施的准确选取与实 施提供了价值依据。学生学习不仅仅是要应付考试, 更 重要的是能够将所学知识应用到实践生活中, 因此在教 学中如果教师能准确地应用绩效技术过程模型分析指导 自己的教学, 灵活运用教学方法, 变换教学方式, 不断 激发学生的学习兴趣, 必然能够促进学生的有效学习。 


\section{5. 绩效技术环境下教师教学的对策}

针对前面提到的小学数学教学中存在的问题, 并结合 绩效技术的理论思想, 笔者对小学教师教学提出以下对策:

\section{1. 多开展活动教学}

目前教师的教学形式比较单一, 局限于课堂教学, 教师讲授, 学生听的模式。为了丰富课堂形式, 可以开 展活动教学的形式。例如可以将课程内容分为两大部分, 一部分为课堂内容, 一部分为实践内容。以“面积”一节内 容为例, 首先是课上内容, 要先回顾已经学过的关于矩 形特征和周长的相关知识, 从而便于新知识的学习, 使 学生建立新旧知识之间的联系; 然后学习本节的新内容, 矩形面积的计算, 并解决相关习题, 同时让学生了解相 关问题的变式, 学会从不同的角度用解决同样的问题; 再让学生进行实践操作, 具体情况是给学生一间大约 10 平方米的小教室, 让学生通过估测并进行相应测量, 确 定张贴的面积, 然后测量并计算每张壁纸的面积, 算出 所需的壁纸数量, 学生进行张贴; 最后任务完成后, 与 大家分享自己小组的学习成果, 交流学习心得, 包括其 中遇到的问题以及后来的解决方案等。通过与实践活动 结合的方式, 让学生更有效的学习。

\section{2. 将理论知识与实际生活相联系}

其实在小学数学教材中经常会出现一些与实际生活 中的事物不相符的情况, 因此需要教师去更正和变通, 将教材内容与实际生活相联系, 为学生创设一个良好的 学习环境, 让学生感觉自己不是在教室学习, 而是在体 验生活, 了解生活。Cheung 曾指出探究中提出的问题实 际上和学生的生活缺乏联系, 教师难以找到探究材料 [8]。 因此, 教师在设计探究的问题时需要将知识点与学生的 现实生活相联系, 从学生的实际生活中获取教学素材, 不断启发和引导学生, 让学生感受到数学中的概念和规 律是从现实生活中抽象出来的[9]。通过这种方式让学生 学会发现和探索生活中的数学问题, 让他们知道其实数 学就在我们身边, 在我们每天的生活中, 让他们在这种 放松的环境中让快乐学习, 既可以培养学生的学习兴趣, 又可以提高学生的学习效率。这就要求教师能够转变自 己的教学理念和态度, 运用自己的教学经验和技巧, 把 各知识巧妙的串联起来帮助学生认知和理解, 以此促进 学生的有效学习。

\section{3. 实施多元评价}

教师检验自己教学成果的万能方式就是通过学生的 考试成绩, 学生考得好, 教师就片面认为是自己教的好; 学生考得差, 教师就以此为辱。这种只要终结性评价, 不要过程性评价的方式是有缺陷的。教师的教学过程是 决定教学结果的直接因素, 也是最重要的因素, 教师对 教学内容的组织与实施、对课堂的掌控与管理、对学生 的提问以及反馈等都可以直接或间接的影响学生的学习
态度、知识获取与理解应用等。因此, 对教师教学的评 价可以从教师的教学态度、教学方法、教学技能、教学 成果等多方面进行。教师要及时地根据学生的课上表现 来反思和改进自己的教学, 从学生身上发现自己的问题, 改进和完善自己的教育教学。

\section{6. 结论}

从专业发展的角度看, 绩效技术起源于教学设计, 且是对教学设计实践领域的扩展, 是对教学系统设计的 一个补充, 但它不能代替教学设计, 指导教学 [10]。绩效 技术是在已有的教学设计的基础上进行的应用研究, 本 研究是从绩效技术的视角, 探究小学数学教学中存在的 问题, 并利用绩效技术过程模型进行教学模式的探究, 以提出相应的问题解决对策。由于绩效技术可以将各个 因素和原因相互关联起来，因此可以帮助教师发现问题， 寻找问题的根本原因并制定相应的解决对策, 从而实现 更好的教学。

\section{参考文献}

[1] 马宁, 林君芬, 林涛, 等. 绩效技术的理论渊源与研究领 域[J]. 中国电化教育, 2004,(10):5-9。

[2] 张祖忻. 从教学设计到绩效技术 $[\mathrm{J}]$. 中国电化教育, 2002,(7):5-8。

[3] 蒋立兵, 余艳. 绩效技术在教学设计中的应用初探 [J]. 远程 教育杂志，2006,(1):61-63。

[4] Deterline, Rosenberg. Workplace Productivity: Performance Technology Success Stories [M]. Washington, DC: International Society for Performance Improvement, 1992.

[5] 潘洪涛, 黎加厚. 绩效技术支持下的教师信息技术培训模 式初探一以上海市浦光中学研究型教师培训为例 [J]. 中小 学电教, 2005,(11):13-15。

[6] 林涛, 马宁, 林君芬, 何克抗. 关于绩效技术的模型评述 [J]. 中国电化教育, 2004,(11):11-15。

[7] 蔡立炉, フ永锋. 高校教师教育技术校本培训的绩效模型 研究[J]. 教育信息化，2006,(10):48-50。

[8] Cheung, D. Facilitating Chemistry Teachers to Implement Inquiry-Based Laboratory Work [J]. International Journal of Science and Mathematics Education, 2008, 6(1): 107-130.

[9] 魏雪峰, 崔光佐, 徐连荣. 基于认知过程分析的小学数学 探究问题设计与应用研究 [J]. 电化教育研究, 2014,35(08):101-107。

[10] 徐点, 孙龙. 基于绩效技术的信息化教学设计与应用研究 [J]. 软件导刊(教育技术), 2014,13(04):13-15。 